incident psoriasis were significantly lower in the DPP4i group versus sulfonylurea with the PS-stratified HR of 0.422 ( $95 \% \mathrm{Cl} 0.273$ to 0.716 ).

Conclusion: DPP4i plus metformin was associated with a reduced risk of psoriasis than sulfonylurea plus metformin. These findings merit further investigation.

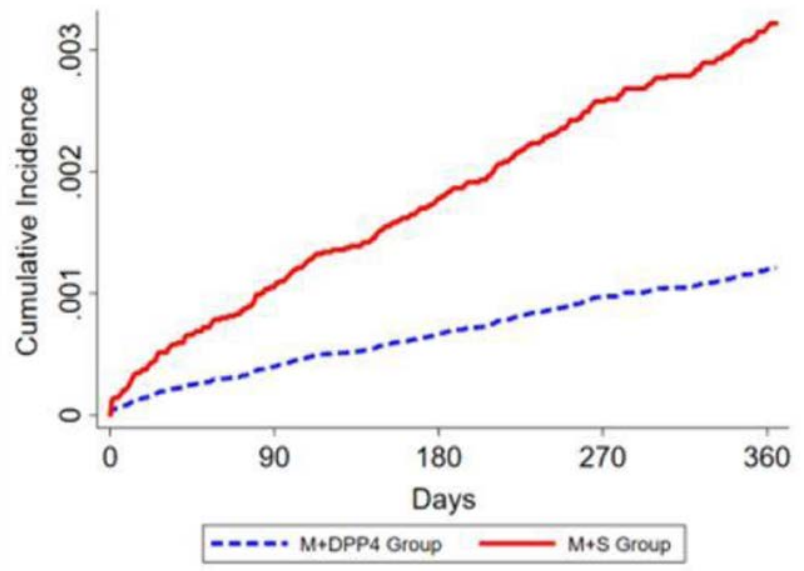

Cumulative Incidence Curve of M+DPP4 Group and M+S Group

Disclosure of Interests: None declared

DOI: 10.1136/annrheumdis-2020-eular.4538

\section{SAT0584 SPECIFIC ACPA REACTIVITIES AND INFLAMMATORY BIOMARKERS ALONG WITH ULTRASOUND TENOSYNOVITIS ARE ASSOCIATED WITH ARTHRITIS ONSET IN A POPULATION AT RISK FOR RHEUMATOID ARTHRITIS}

A. Circiumaru ${ }^{1}$, Y. Kisten ${ }^{1}$, M. Hansson ${ }^{1}$, V. Joshua ${ }^{1}$, M. Sun ${ }^{1}$, H. Rezaei ${ }^{1}$, E. Af Klint ${ }^{1,2}$, A. Antovic ${ }^{1,3}$, A. Catrina ${ }^{1,2,3}$, A. Hensvold ${ }^{1,3} .{ }^{1}$ Rheumatology unit, Department of Medicine, Karolinska Institutet, Stockholm, Sweden ${ }^{2}$ Rheumatology clinic, Karolinska University Hospital, Stockholm, Sweden; ${ }^{3}$ Center for Rheumatology, Academic Specialist Center, Stockholm Health Region, Stockholm, Sweden

Background: Anti-citrullinated protein antibodies (ACPA) are characteristic markers for rheumatoid arthritis (RA), developing years before disease onset. Early clinical and biological biomarkers could provide useful information on the onset of RA in predisposed individuals.

Objectives: The aim of the study was to investigate whether ACPA along with inflammatory markers and musculoskeletal ultrasound changes could predict arthritis development in individuals at risk for RA.

Methods: ACPA-positive individuals with musculoskeletal complaints were referred from primary care to a rheumatology clinic, recruited in the Risk-RA research program and followed-up for up to 3 years, between April 2014 and October 2019. All individuals lacked arthritis both at clinical examination by a trained rheumatologist and ultrasound assessment of hands and feet and any other symptomatic joints (according to EULAR-OMERACT definition). Blood samples were collected at inclusion and were analyzed for 15 ACPA fine specificities (by custom made peptide array), 92 inflammation-associated protein biomarkers (by multiplex immunoassay with Olink extension technology) and HLA-SE (DR low resolution kit). Statistical analysis used univariate and multivariate models with backwards selection and cox regression.

Results: 268 individuals with a median age of $48(36-58)$ were recruited, out of which $212(79 \%)$ were females. $75(28 \%)$ developed arthritis within 11 months of follow-up while the median follow-up for those not developing arthritis was 21 months (14-28). Increased ACPA levels, shorter symptom duration and RF positivity were the main differences between individuals developing arthritis and those who did not. In univariate models, the presence of HLA-SE, specific ACPA reactivities, certain inflammatory markers and ultrasound-detected tenosynovitis were associated with arthritis development. In multivariate analysis the presence of anti-cit-fillagrin (HR 2.1 (95\% Cl 1.2-3.7, p 0.01), IL6 levels (HR $1.4(95 \% \mathrm{Cl}$ $1.2-1.7, \mathrm{p} 0.0001)$ and tenosynovitis (HR $2.9(95 \% \mathrm{Cl}$ 1.7-5.0, p 0.0001) remained significant predictors for arthritis onset.

Conclusion: Certain ACPA reactivities together with inflammatory markers and ultrasound-detected tenosynovitis predict arthritis development in predisposed individuals for developing RA.
Disclosure of Interests: None declared

DOI: 10.1136/annrheumdis-2020-eular.5578

\section{SAT0585 GEO-EPIDEMIOLOGY OF AUTOANTIBODIES IN RA: DIFFERENT PREVALENCES IN FOUR ETHNICALLY DIVERSE RA POPULATIONS}

E. C. De Moel ${ }^{1}$, V. Derksen ${ }^{1}$, L. A. Trouw ${ }^{1}$, C. Terao ${ }^{2}$, M. Tikly ${ }^{3}$, H. El-Gabalawy ${ }^{4}$, H. Bang ${ }^{5}$, T. Huizinga ${ }^{1}$, R. Toes ${ }^{1}$, D. Van der Woude ${ }^{1} .{ }^{1}$ Leiden University Medical Center (LUMC), Leiden, Netherlands; ${ }^{2}$ Kyoto University Graduate School of Medicine, Genomic Medicine, Kyoto, Japan; ${ }^{3}$ University of the Witwatersrand, Division of Rheumatology, Johannesburg, South Africa; ${ }^{4}$ University of Manitoba, Department of Internal Medicine, Winnipeg, Canada; ${ }^{5}$ Orgentec Diagnostika GmbH, Mainz, Germany

Background: Rheumatoid arthritis (RA) has been described in virtually every ethnic population. Most RA patients harbor anti-modified protein antibodies (AMPAs), including anti-citrullinated protein (ACPA), anti-carbamylated protein (anti-CarP), anti-malondialdehyde acetaldehyde (anti-MAA), and anti-acetylated protein antibodies (AAPA). However, it is unclear whether differences exist in the AMPA response between different ethnic groups. Such differences could provide new clues to genetic and environmental factors contributing to autoantibody development

Objectives: To investigate the prevalence of different AMPA in four ethnically diverse RA populations, and their association with smoking

Methods: Enzyme-linked immunosorbent assays were used to measure antiCarP IgG, anti-MAA IgG (both in-house), and anti-acetylated vimentin IgG (Orgentec) in ACPA-positive sera of Dutch (NL, $n=103$ ), Japanese (JP, $n=174$ ), Canadian First Nations People (FN, $n=100$ ), and black South Africans ( $\mathrm{A} A, n=67$ ) fulfilling the 1987 ACR classification criteria for RA. Ethnicity-matched local healthy controls were used to calculate cohort-specific cut-offs. Logistic regression was used to identify whether ever-smoking was associated with AMPA seropositivity in each cohort, corrected for age, gender, and disease duration. Random-effects meta-analysis was used to pool the resulting odds ratios (OR). Results: For all three AMPAs, median levels were higher in FN and especially SA than NL and JP patients (Figure 1). The median autoantibody levels in arbitrary units (in \% of patients positive) for NL, JP, FN and SA RA patients were: antiCarP IgG: 1157 (47\%), 994 (43\%), $1642(58 \%)$ and 2336 (76\%) (p<0.001); antiMAA IgG: 131 (29\%), $179(22 \%), 251(29 \%)$ and 257 (53\%) ( $<<0.001)$; AAPA: $133(20 \%), 136(17 \%), 153(38 \%)$ and $316(28 \%)(p<0.001)$. Prevalence, meaning positivity, also differed significantly between cohorts for all AMPAs $(p<0.001)$. There were also marked differences in total IgG levels in mean (SD) $g / L: 13$ (4) for NL, 17 (6) for JP, 18 (6) for FN, and 25 (8) for SA ( $p<0.001)$. When the autoantibody levels were normalized to total $\lg G$, the differences in became less pronounced between cohorts (Figure 2). The median arbitrary units per $\mathrm{g} / \mathrm{L}$ Total IgG for NL, JP, FN and SA RA patients were: anti-CarP IgG: 54, 25, 53, and 79, anti-MAA IgG: 6, 5, 8, and 9; and AAPA: 2, 2, 2, and 3, suggesting that autoantibody level differences may partly correspond to cohort-specific differences in total IgG, although the overall trend of higher levels in SA persisted. There was no association between smoking and anti-CarP or anti-MAA positivity, with pooled OR $(95 \% \mathrm{Cl})$ of $1.31(0.79-2.18)$ and 0.85 (0.46-1.56), respectively. However, smoking was positively and consistently associated with AAPA positivity in each cohort: pooled OR $(95 \% \mathrm{Cl})$ of 2.01 (1.06-3.81)

Conclusion: In these ACPA-positive ethnically diverse RA populations, levels and prevalence of various AMPAs differ, suggesting that ethnic background and environment may influence the development of the autoantibody response in RA. Despite these differences, our results imply smoking as a consistent risk factor for AAPA across different ethnic backgrounds.

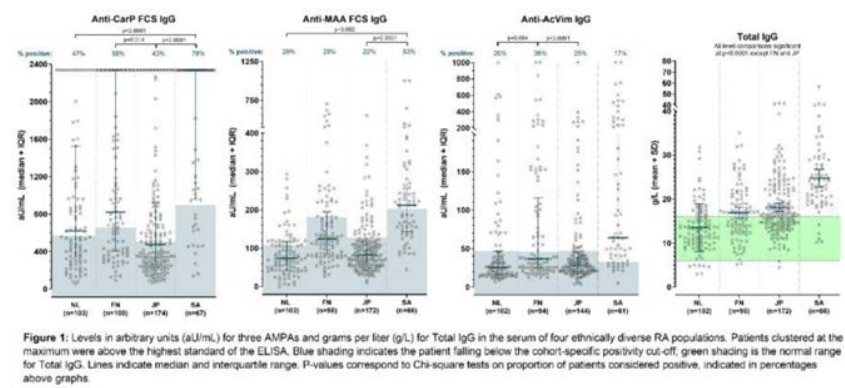



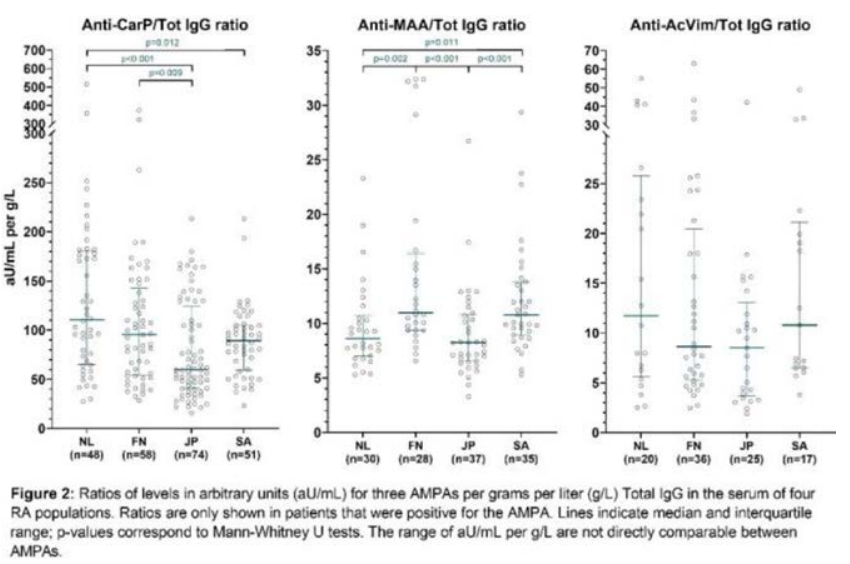

RA populations. Ratios are only shown in patients that were positive for the AMPA. Lines indicate median and interquartile range; p-values correspond to Mann-Whitney $\mathrm{U}$ tests. The range of $\mathrm{aU} / \mathrm{mL}$ per $\mathrm{g} / \mathrm{L}$ are not directly compar

Disclosure of Interests: Emma C. de Moel: None declared, Veerle Derksen: None declared, Leendert A Trouw: None declared, Chikashi Terao: None declared, Mohammed Tikly: None declared, Hani El-Gabalawy: None declared, Holger Bang Grant/research support from: Employee of Orgentec Diagnostika, Thomas Huizinga Grant/research support from: Ablynx, Bristol-Myers Squibb, Roche, Sanofi, Consultant of: Ablynx, Bristol-Myers Squibb, Roche, Sanofi, Rene Toes: None declared, Diane van der Woude: None declared

DOI: 10.1136/annrheumdis-2020-eular.3146

\section{SAT0586 PREVALENCE AND RISK FACTORS FOR CARDIO- METABOLIC ABNORMALITIES IN PATIENTS WITH INFLAMMATORY ARTHRITIS ATTENDING CARDIO- RHEUMATOLOGY PRIMARY PREVENTION CLINICS}

L. Eder ${ }^{1}$, S. Akhtari', P. Harvey ${ }^{1}$, K. Bindee ${ }^{2} .{ }^{1}$ Women's College Hospital, University of Toronto, Toronto, Canada; ${ }^{2}$ Sinai Health System, University of Toronto, Toronto, Canada

Background: Cardio-metabolic abnormalities are common in patients with inflammatory arthritis (IA) but tend to be under-recognized and under-treated. Objectives: We aimed to compare the prevalence and risk factors for cardio-metabolic abnormalities between patients with rheumatoid arthritis (RA), psoriatic arthritis (PsA) and ankylosing spondylitis (AS).

Methods: Consecutive patients enrolled in the University of Toronto Cardio-Rheumatology Network from July 2017 to August 2019 were analyzed. This is a primary prevention program that uses structured clinical, laboratory and multimodal imaging to diagnose and treat cardiovascular disease (CVD). Patients with a rheumatologist-confirmed diagnosis of RA, PsA or AS with no known CVD were evaluated. Information about IA diagnosis, medications and comorbidities was recorded. Each patient was evaluated by a cardiologist focusing on CVD risk assessment. We evaluated the prevalence of previously recorded and newly recognized cardio-metabolic risk factors including hypertension, dyslipidemia, obesity and diabetes. The prevalence of these abnormalities was compared between IA diagnoses. Regression models were used to assess the association between diagnosis and cardio-metabolic abnormalities after adjusting for demographics, smoking, BMI, measures of disease activity and medications.

Results: A total of 358 patients (201 RA, 124 PsA, 33 AS) were assessed (mean age $59 \pm 10.5$ years, $68.7 \%$ female). Hypertension was reported in $33 \%$, dyslipidemia in $26.8 \%$, diabetes mellitus in $8.9 \%$ and overweight/obesity in $69.7 \%$ (Figure 1). Newly detected elevations in lipids were frequent for triglycerides (9.3\%), non-HDL-cholesterol (6\%,) and LDL-cholesterol (2.7\%). Elevated HbA1c occurred in $1.4 \%$ and newly diagnosed hypertension occurred in $9.8 \%$. A total of $32.8 \%$ patients required a change or initiation of medications for their cardio-metabolic abnormalities (21.7\% lipid-lowering therapy, $14.6 \%$ aspirin, $11.1 \%$ anti-hypertension therapy). Patients with PsA had the highest prevalence of cardio-metabolic abnormalities including dyslipidemia, obesity and hypertension. Having hypertension (prior or new diagnosis), elevated levels of triglycerides, non-HDL cholesterol, total cholesterol and BMI were associated with PsA vs. RA after adjusting for potential confounders (all $\mathrm{p}<0.05$ ) (Figure 2). No significant association was found between cardio-metabolic abnormalities and AS vs. PsA or RA.

Conclusion: Dedicated cardio-rheumatology clinics have improved CVD screening and management in an IA population. The burden of cardio-metabolic abnormalities is elevated in PSA and suggests that tailored strategies to reduce adverse CVD events are particularly needed in this subgroup.
Figure 1 - The Prevalence of Cardiovascular Risk Factors (Previously diagnosed and Newly diagnosed)
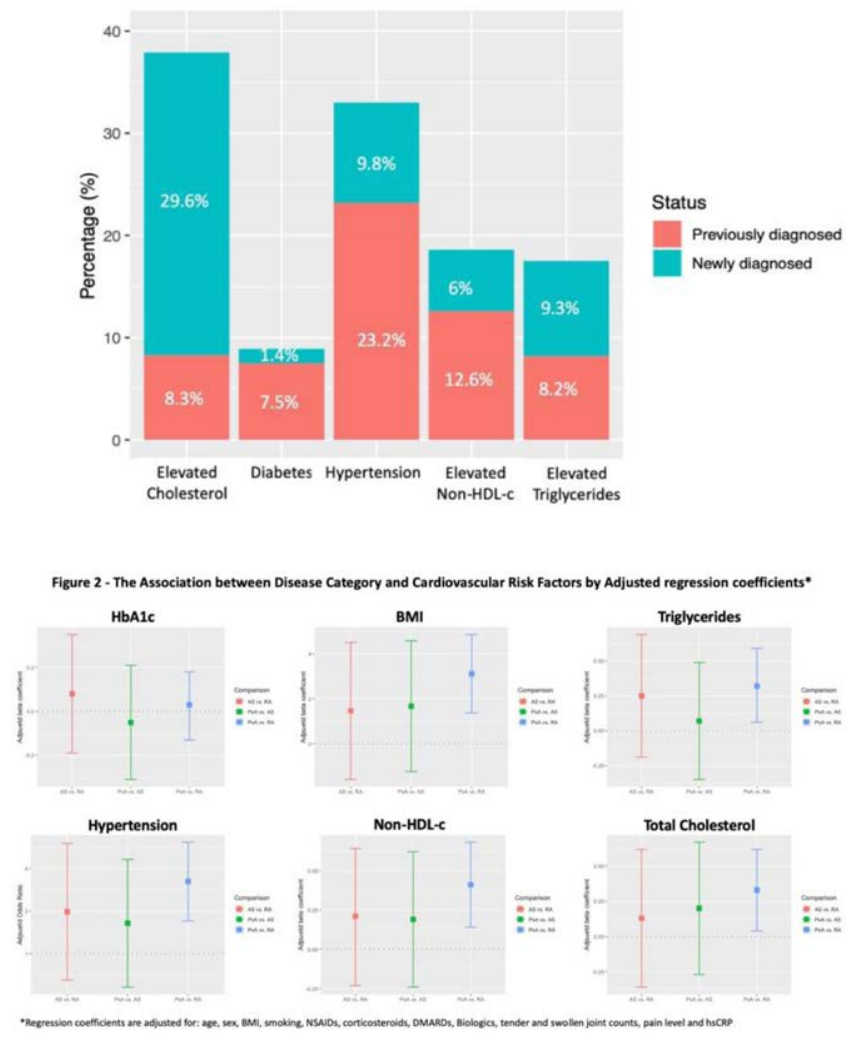

Disclosure of Interests: Lihi Eder Grant/research support from: Abbvie, Lily, Janssen, Amgen, Novartis, Consultant of: Janssen, Speakers bureau: Abbvie, Lily, Janssen, Amgen, Novartis, Shadi Akhtari: None declared, Paula Harvey: None declared, Kuriya Bindee Grant/research support from: Abbvie, Pfizer Sanofi, BMS, Consultant of: Abbvie, Eli Lily, Pfizer DOI: 10.1136/annrheumdis-2020-eular.1228

\section{SAT0587 MACHINE-LEARNING DERIVED ALGORITHMS FOR OUTCOMES PREDICTION IN RHEUMATIC DISEASES: APPLICATION TO RADIOGRAPHIC PROGRESSION IN EARLY AXIAL SPONDYLOARTHRITIS}

R. Garofoli ${ }^{1}$, M. Resche-Rigon ${ }^{2}$, M. Dougados ${ }^{1}$, D. Van der Heijde ${ }^{3}$, C. Roux ${ }^{1}$, A. Moltó ${ }^{1}{ }^{1}$ Hospital Cochin, Rheumatology, Paris, France; ${ }^{2}$ Hospital Saint-Louis Ap-Hp, Paris, France; ${ }^{3}$ Leiden University Medical Center, Leiden, Netherlands

Background: Axial spondyloarthritis (axSpA) is a chronic rheumatic disease that encompasses various clinical presentations: inflammatory chronic back pain, peripheral manifestations and extra-articular manifestations. The current nomenclature divides axSpA in radiographic (in the presence of radiographic sacroilitis) and non-radiographic (in the absence of radiographic sacroiliitis, with or without MRI sacroiliitis. Given that the functional burden of the disease appears to be greater in patients with radiographic forms, it seems crucial to be able to predict which patients will be more likely to develop structural damage over time. Predictive factors for radiographic progression in axSpA have been identified through use of traditional statistical models like logistic regression. However, these models present some limitations. In order to overcome these limitations and to improve the predictive performance, machine learning (ML) methods have been developed.

Objectives: To compare ML models to traditional models to predict radiographic progression in patients with early axSpA.

Methods: Study design: prospective French multicentric cohort study (DESIR cohort) with 5years of follow-up. Patients: all patients included in the cohort, i.e. 708 patients with inflammatory back pain for $>3$ months but $<3$ years, highly suggestive of axSpA. Data on the first 5 years of follow-up was used. Statistical analyses: radiographic progression was defined as progression either at the spine (increase of at least 1 point per 2 years of mSASSS scores) or at the sacroiliac joint (worsening of at least one grade of the mNY score between 2 visits). Traditional modelling: we first performed a bivariate analysis between our outcome (radiographic progression) and explanatory variables at baseline to select the variables to be included in our models and then built a logistic regression model (M1). Variable selection for traditional models was performed with 2 different methods: stepwise selection based on 